\title{
Estudo de propriedades físico-químicas na avaliação da adsorção em biocarvão produzido com folhas de amendoeira-da-praia
}

\author{
Study of physico-chemical properties in the evaluation of adsorption in \\ biochar produced from leaves of amendoeira-da-praia
}

\begin{abstract}
Carolina Carvalho de Souza' ${ }^{\oplus}$, Katia Noriko Suzuki'* ${ }^{\oplus}$, Rose Mary Latini' ${ }^{\oplus}$, Fatima de Paiva Canesin $^{1} \odot$, Ana Maria Rangel de Figueiredo Teixeira' ${ }^{\circledR}$, Alfredo Victor Bellido Bernedo ${ }^{1} \odot$
\end{abstract}

\section{RESUMO}

Este estudo avaliou as propriedades físico-químicas envolvidas no processo de adsorção para subsidiar o potencial uso de biocarvão produzido com folhas secas de amendoeira-da-praia, Terminalia catappa, como remediador em águas contaminadas com metais. O biocarvão utilizado foi produzido por conversão à baixa temperatura e caracterizado por apresentar bandas de O-H, $\mathrm{CH}_{2}$, C-O e C-O-C; teores de 48,8\% de carbono, 46,6\% de oxigênio, 3,3\% de hidrogênio e demais entre enxofre e nitrogênio; presença de macroporos e passado em peneira (100 mesh). Para o estudo, preparou-se um coquetel de radiotraçadores (Co-57, Mn-54 e Zn-65), que foi adicionado à água deionizada e massas de biocarvão com o objetivo de obter parâmetros tais como pH, tempo de equilibrio e capacidade de adsorção por meio das isotermas de Langmuir e Freundlich. O detector de Germânio Hiperpuro foi utilizado para quantificar as atividades dos radiotraçadores por meio da técnica de espectroscopia gama para geração e tratamento de dados. Os resultados obtidos mostraram aumento na adsorção do pH 3,8 em diante e tempo de equilíbrio de 30 minutos ou mais. As isotermas de Langmuir e Freundlich construídas apresentaram bons ajustes, com valores de n maiores que 1, indicando preferência dos metais pelo material adsorvente. Além disso, levando-se em conta a competição pelos sítios ativos e a concentração inicial das soluções (ordem de picogramas), foram encontradas capacidades de adsorção de 1,273 ng.g-1 para o Zn-65, de 3.271 ng.g-1 para o Co-57 e de 9,026 ng.g-1 para o Mn-54.

Palavras-chave: biocarvão; cinética de adsorção; radiotraçadores; isotermas; remediação.

\begin{abstract}
This study evaluated the physical-chemical properties of the adsorption process in biochar produced from dry leaves of beach-almond, Terminalia catappa, as a useful tool for remediation purposes in water contaminated with metals. The biochar was characterized by having: bands of $\mathrm{O}-\mathrm{H}, \mathrm{CH}_{2}, \mathrm{C}-\mathrm{O}$, and C-O-C; contents of $48.8 \%$ carbon, $46.6 \%$ oxygen, $3.3 \%$ hydrogen and the others between sulfur and nitrogen; the presence of macropores; and being sieved (100 mesh). Parameters such as pH, equilibrium time and adsorption capacity by the Langmuir and Freundlich isotherms were determined from experiments using a radiotracer cocktail (Co-57, Mn-54 and Zn-65) which was added to deionized water and a constants mass of biochar. The high-purity germanium detector (HPGe) was used to quantify the radiotracer activity by gamma spectroscopy. The results showed better adsorption from $\mathrm{pH} 3.8$ and 30 minutes equilibrium time. The Langmuir and Freundlich isotherms had a good model fit, with $n$ values higher than 1 , and showed affinity between the metals and the biochar. The adsorption capacities of Zn-65, Co-57 and Mn-54

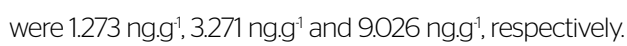

Keywords: biochar; kinetics adsorption; radiotracers; isotherms; remediation. 


\section{INTRODUÇÃO}

A introdução de metais por ação antrópica em quantidades acima do natural é observada em todo o mundo. A presença de metais na água e em solos, dependendo de sua dose e forma química, pode afetar todas as formas de vida. Seus efeitos tóxicos no homem podem ser considerados agudos e de curto prazo, quando são mais evidentes (como, por exemplo, na diarreia), ou ter abrangência de médio e longo prazo, quando são menos evidentes, difíceis de serem distinguidos e inespecíficos, pela possibilidade de terem sido provocados por outras substâncias ou interações entre os metais. A manifestação desses efeitos dá-se especialmente pela dosagem do metal e pode se espalhar por todo o corpo, alterando o metabolismo. Como exemplo, podemos citar tanto o cobalto quanto o manganês e o zinco, definidos como elementos essenciais e simultaneamente contaminantes, ou seja, em doses elevadas apresentarão efeitos nocivos (KAWAI et al., 2017).

De maneira geral, ao aumento da poluição está associada a busca por alternativas sustentáveis de remediação. Grande parte dos efluentes industriais e domésticos apresenta poluentes metálicos, os quais se acumulam na biota e têm efeitos nocivos ao organismo, como mencionado. Desse modo, requer-se que o efluente seja tratado, para a segurança dos recursos hídricos e de todo o ambiente à sua volta. Uma dessas opções é o uso de biocarvões, os quais podem ser obtidos por processos de pirólise de conversão à baixa temperatura (CBT) de uma biomassa. Sua aplicação como material adsorvente vem sendo amplamente estudada em função do seu poder de adsorção, uma vez que no processo de conversão da biomassa se busca obter materiais com melhores propriedades físico-químicas. Entre essas propriedades está o poder de adsorção do biocarvão, associado intimamente às forças de London ou de Van der Waals envolvidas entre sua superfície porosa e as moléculas do fluido a ser tratado (RORIS et al., 2008). O uso de radiotraçadores apresenta-se como um diferencial, já que permite o estudo dessas propriedades físico-químicas mesmo em baixa concentração, o que nem sempre é possível com metais estáveis (BELLIDO et al., 2013).

O artigo $6^{\circ}$ da Resolução do Conselho Nacional do Meio Ambiente (CONAMA) no 420/2009 define remediação como "uma das ações de intervenção para reabilitação de área contaminada, que consiste em aplicação de técnicas, visando à remoção, contenção ou redução das concentrações de contaminantes" (BRASIL, 2009). Assim, podemos enquadrar o biocarvão como um remediador por sua capacidade de adsorção. Esta é um dos métodos mais tradicionais para a remoção de quantidades traço de contaminantes que nem sempre são passíveis de tratamento por degradação biológica ou outros métodos físico-químicos e vem sendo estudada por sua aplicabilidade em diversos setores, tais como: o tecnológico, o biológico, o industrial e o de proteção ambiental (NASCIMENTO et al., 2014).

A adsorção que usa biocarvão produzido com restos de plantas e resíduos agrícolas tornou-se uma alternativa sustentável, eficiente e de baixo custo para a descontaminação de águas contaminadas com poluentes orgânicos e inorgânicos, incluindo poluentes metálicos. Vale ressaltar que a adsorção dos metais não depende exclusivamente das características do biocarvão, mas também da natureza dos metais e seus respectivos comportamentos ao competirem pelos sítios ativos do biocarvão (PARK et al., 2016).

O objetivo deste estudo foi avaliar as propriedades físico-químicas envolvidas em um processo de adsorção de metais, para subsidiar o potencial uso de biocarvão produzido com folhas secas de amendoeira-da-praia, Terminalia catappa, como remediador em águas contaminadas com metais. Para tal, empregou-se um coquetel que continha, simultaneamente, os radiotraçadores Co-57, Mn-54 e Zn-65, que são alguns dos metais presentes em águas contaminadas. A escolha pelo uso de um coquetel contendo três metais deve-se ao fato de que, na prática, os efluentes e águas a serem tratados, em geral, não apresentam apenas um contaminante metálico. Além de serem bastante comuns em nossa região, as folhas de amendoeira-da-praia apresentaram bons resultados para a produção de biocarvão, conforme estudos realizados por Souza e Teixeira (2015), ao testarem diferentes rejeitos urbanos.

\section{METODOLOGIA}

O biocarvão escolhido para o presente estudo foi produzido com uma biomassa de folhas secas de amendoeira-da-praia coletadas no município de São Gonçalo (RJ). Essa biomassa foi então transferida para uma caixa metálica com entrada e saída de gás, a qual foi saturada com atmosfera de gás carbônico e colocada em uma mufla (EDG Equipamentos, modelo EDG10P-5). À saída de gases, foi acoplado um condensador para a condensação dos voláteis constituintes do gás, da água e do óleo da CBT (Figura 1).

Os patamares de temperatura utilizados na conversão foram, em 30 minutos, $25,180,240$ e $350^{\circ} \mathrm{C}$, respectivamente, o que constitui conversão à

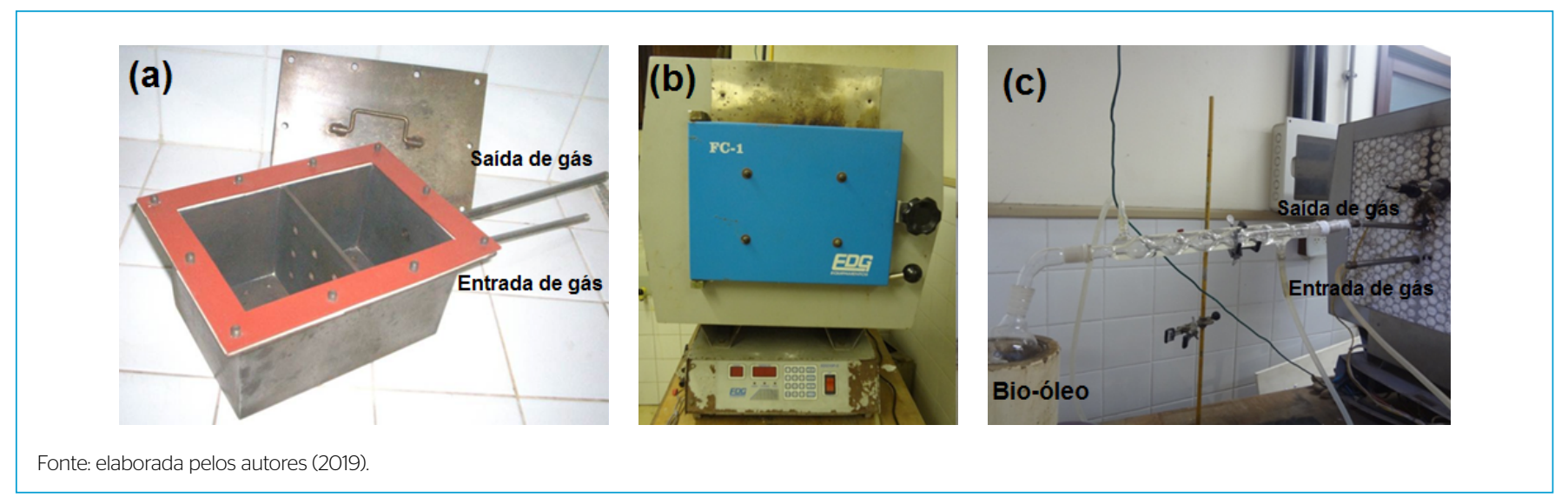

Figura 1 - (A) Caixa metálica com entrada e saída de gás; (B) mufla (EDG Equipamentos, modelo EDG1OP-5; (C) condensador acoplado à caixa para produção do biocarvão. 
baixa temperatura. Após a CBT, o biocarvão foi retirado da caixa metálica e os voláteis condensados ficaram no balão acoplado ao condensador. O biocarvão foi então peneirado em peneira de 100 mesh (SOUZA; TEIXEIRA, 2015). Após a produção do biocarvão, realizou-se o estudo de suas propriedades físico-químicas, seguido da identificação de alguns parâmetros para a sua caracterização.

\section{Estudo das propriedades físico-químicas}

Para a análise das propriedades físico-químicas envolvidas no processo de adsorção de metais em solução aquosa, preparou-se um coquetel radioativo com os radiotraçadores Co-57, Mn-54 e Zn-65; determinou-se o pH ideal e o tempo de equilíbrio de adsorção e construíram-se as isotermas de adsorção. Os experimentos foram realizados em batelada. Em todas essas etapas, a atividade dos radiotraçadores foi determinada por espectrometria gama. Esta é uma técnica analítica quantitativa e qualitativa baseada no princípio de que cada radioisótopo emite radiação gama em energias características, permitindo sua identificação e a quantificação da sua atividade pela análise de um espectro de radiação gama, além da detecção simultânea de radioisótopos (KNOLL, 2011). No presente trabalho, os espectros de radiação gama foram analisados pelo software Genie 2000, versão 3.2.1/2009. A Figura 2 apresenta um esquema do funcionamento do detector de germânio hiperpuro (HPGe) e eletrônica associada.

O coquetel radioativo, denominado de solução mãe, foi preparado por meio de diluições de soluções comerciais dos diferentes radiotraçadores, fornecidas pela PerkinElmer Ltda. em HCl 0,5 M. As características dos radiotraçadores estão descritas na Tabela 1. Toda a parte experimental foi realizada no Laboratório de Química Nuclear e Radioquímica da Universidade Federal Fluminense. As medidas das atividades dos radiotraçadores antes e depois do contato com o biocarvão em estudo foram realizadas no Laboratório de Radioisótopos Aplicados ao Meio Ambiente (LARAMAM/UFF), utilizando um detector de HPGe, marca Canberra, modelo GX4021, o qual é refrigerado por nitrogênio líquido a $-196^{\circ} \mathrm{C}$ e opera a $2,500 \mathrm{~V}$.

$\mathrm{Na}$ espectrometria gama, para garantir melhor estatística nas contagens, foram realizadas medidas de erro de contagem abaixo de $1 \%$ e tempo morto menor que 10\%, (BELLIDO; LATINI, 2014). Foram também efetuadas correções do decaimento radioativo, em razão da diferença de tempo entre os experimentos e a contagem das amostras, considerando-se o tempo de meia-vida dos radiotraçadores (BELLIDO et al., 2013; SONDERMANN, 2015). O presente trabalho considerou a eficiência intrínseca para a apresentação de seus resultados, a qual foi calculada com base em um padrão sólido de Eu-152 fornecido pelo Instituto de Radioproteção e Dosimetria (IRD). Após a correção pela eficiência, as atividades até então dadas em contagens por segundo foram convertidas para bequerel (Bq). Posteriormente, fez-se a conversão de Bq para grama, uma vez que os resultados da capacidade de adsorção (q) serão apresentados em g.g-1 (BRYAN, 2013).

Com a solução mãe pronta, passou-se para a etapa seguinte, que foi o experimento para a determinação do $\mathrm{pH}$ ideal de adsorção. Para a construção da curva de $\mathrm{pH}$ em função do percentual de remoção dos radiotraçadores, foram preparadas soluções de diferentes $\mathrm{pH}$, entre 2 e 8 , com água deionizada (SOUZA; TEIXEIRA, 2015), ajustadas com soluções diluídas de ácido clorídrico e hidróxido de amônio e medidas no pHmetro Hanna Instruments. A essas soluções foram acrescentados os radiotraçadores e, para que não houvesse mudança do seu $\mathrm{pH}$, inicialmente, alíquotas da solução mãe foram colocadas em frasco acrílico de $20 \mathrm{~mL}$ e secas em estufa a $63^{\circ} \mathrm{C}$ por 1 a 2 horas até a evaporação da solução. Após a secagem, os radiotraçadores foram ressuspensos na solução com o pH desejado. Em seguida, toda a solução foi transferida para uma seringa plástica de $10 \mathrm{~mL}$ contendo $20 \mathrm{mg}$ de biocarvão previamente pesados em balança analítica e posteriormente homogeneizados em agitador mecânico com movimento horizontal (100 rpm) durante 1 hora. Após o tempo de contato, as soluções foram filtradas manualmente em membrana descartável estéril de 0,22 $\mu \mathrm{m}$ (Millipore, PVDF, $25 \mathrm{~mm}$ de $\varnothing$ ). A Tabela 2 mostra as concentrações iniciais (pg.L-1 $)$ para os radiotraçadores Co-57, Mn-54 e Zn-65 utilizados no experimento.

Foi feito também um "branco", cuja seringa não apresentou biocarvão, e que permitiu quantificar os radiotraçadores que ficaram adsorvidos nas paredes dos

Tabela 1 - Características dos radiotraçadores Co-57, Mn-54 e Zn-65.
\begin{tabular}{c|c|c|c} 
Radiotraçador & Energia (keV) & $\begin{array}{c}\text { Tempo de meia-vida } \\
\text { (dias) }\end{array}$ & Abundância (\%) \\
\hline Co-57 & 122,06 & 271,80 & 87,53 \\
\hline Mn-54 & 834,85 & 312,19 & 99,98 \\
\hline Zn-65 & $1.115,50$ & 244,10 & 50,23 \\
\hline
\end{tabular}

Fonte: LNHB (2016).

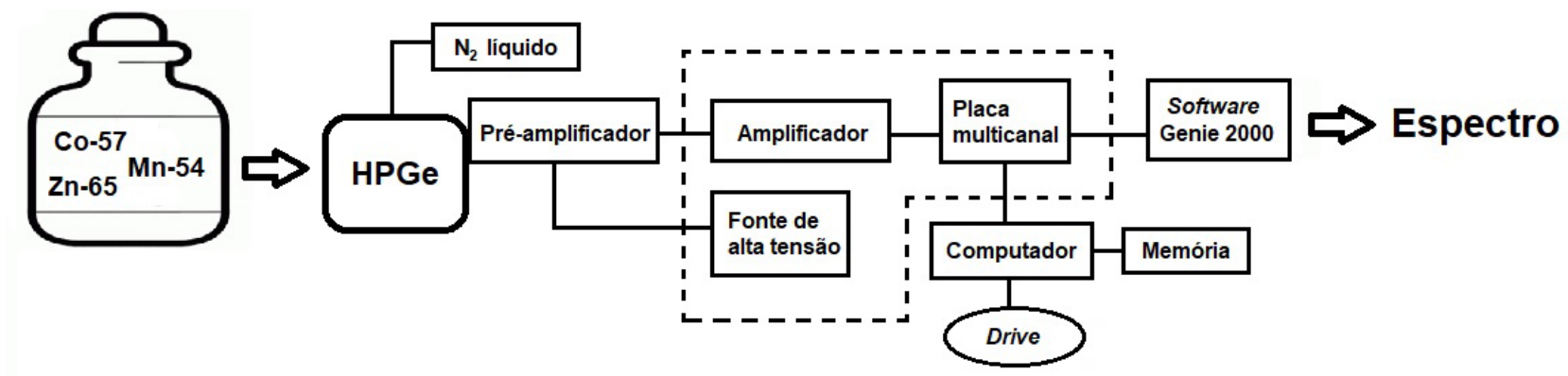

HPGe: germânio hiperpuro.

Fonte: elaborada pelos autores (2019)

Figura 2 - Esquema de funcionamento do detector HPGe e eletrônica associada. 
materiais utilizados (especialmente seringa e filtro) e não no biocarvão. Os valores utilizados no estudo foram corrigidos pelo valor de "branco" obtido. Antes do contato com o biocarvão, foi realizada uma medida da solução ressuspensa no detector de HPGe para determinar a atividade inicial dos radiotraçadores, denominada de (Ai). Após o contato com o biocarvão, realizou-se outra medida para a amostra filtrada (Af). O cálculo da eficiência (\%) de remoção de radiotraçadores pelo biocarvão foi efetuado sobre a diferença entre as atividades iniciais e do filtrado (Equação 1).

Remoção (\%) $=[(\mathrm{Ai}-\mathrm{Af}) / \mathrm{Ai}] \mathrm{x} 100$

Com os dados obtidos, construiu-se uma curva relacionando a eficiência (\%) de remoção dos radiotraçadores (Co-57, Mn-54 e Zn-65) pelo biocarvão em função dos valores de $\mathrm{pH}$, de modo a determinar a influência do $\mathrm{pH}$ no processo de adsorção. Com o pH ideal de adsorção definido no experimento anterior, passou-se para a determinação do tempo de equilíbrio da adsorção, por meio do levantamento de uma curva de diferentes tempos de contato/agitação em função da eficiência (\%) de remoção de radiotraçadores. Para cada tempo de agitação foram feitas duplicatas. A Tabela 3 mostra as concentrações iniciais (pg. $\mathrm{L}^{-1}$ ) para os radiotraçadores utilizados no experimento. O cálculo da eficiência de (\%) remoção foi feito de acordo com a Equação 1.

Com o tempo de equilíbrio definido, a etapa seguinte foi a construção das isotermas de adsorção, na qual se variaram as massas de radiotraçadores. A Tabela 4 mostra as concentrações iniciais (pg.L $\mathrm{L}^{-1}$ ) para os radiotraçadores utilizados no experimento. Fez-se também um "branco", cuja seringa não apresentou biocarvão. Para o cálculo da eficiência (\%) de remoção, utilizou-se a Equação 1.

Após a obtenção e a análise dos dados, foram construídas as isotermas de Langmuir e Freundlich. A capacidade de adsorção em g.g ${ }^{-1}(q)$ foi calculada pela Equação 2:

$\mathrm{q}=(\mathrm{Co}-\mathrm{Ce}) \cdot \mathrm{V} / \mathrm{m}$

Em que:

$\mathrm{V}=\mathrm{o}$ volume da solução na seringa $(\mathrm{L})$;

$C_{o}=$ a concentração inicial de radiotraçador antes do contato com o biocarvão $\left(\right.$ g. $\left.\mathrm{L}^{-1}\right)$;

$C_{e}=$ a concentração de radiotraçador na solução do filtrado após o contato com o biocarvão (g. $\left.\mathrm{L}^{-1}\right)$;

$m=$ a massa de biocarvão (g) (NASCIMENTO et al., 2014).

Para a obtenção da capacidade máxima de adsorção $\left(\mathrm{g} \cdot \mathrm{g}^{-1}\right)$ do radiotraçador por unidade de biocarvão (g), os dados foram modelados pela equação da isoterma de Langmuir (Equação 3):

$\frac{1}{q_{e}}=\frac{1}{q_{\max }}+\frac{1}{K_{L} q_{\max } C_{\epsilon}}$

Em que:

$q_{e}=$ a capacidade de adsorção $\left(\mathrm{g} \cdot \mathrm{g}^{-1}\right)$;

$q_{\max }=$ a capacidade máxima de adsorção $\left(\mathrm{g} \cdot \mathrm{g}^{-1}\right)$ do radiotraçador por unidade de biocarvão (g);

$C_{e}=$ a concentração de radiotraçador na solução do filtrado após o contato com o biocarvão (g.L $\left.\mathrm{L}^{-1}\right)$;
$\mathrm{K}_{\mathrm{L}}=\mathrm{a}$ constante de equilíbrio de Langmuir relacionada à energia de adsorção (L.g $\left.{ }^{-1}\right)$ (NASCIMENTO et al., 2014).

Posteriormente, os dados foram linearizados pelo gráfico $1 / \mathrm{q}\left(\mathrm{g} . \mathrm{g}^{-1}\right) \mathrm{em}$ função de $1 / C_{e}\left(\mathrm{~L} \cdot \mathrm{g}^{-1}\right)$.

A isoterma de Freundlich, por sua vez, foi linearizada pelo gráfico $\log q \mathrm{em}$ função do $\log C_{e^{\prime}}$, cuja equação linear é dada pela Equação 4 :

$\log \mathrm{q}_{\mathrm{e}}=\log \mathrm{K}_{\mathrm{F}}+1 / \mathrm{n} \log \mathrm{C}_{\mathrm{e}}$

Em que:

$q=$ a capacidade de adsorção $\left(\mathrm{g} \cdot \mathrm{g}^{-1}\right)$;

$C_{e}=$ a concentração de radiotraçador na solução do filtrado após o contato com o biocarvão (g.L-1 $)$;

$\mathrm{K}_{\mathrm{F}}=\mathrm{a}$ constante de equilíbrio de Freundlich relacionada à capacidade de adsorção (g.g $\left.\mathrm{g}^{-1}\right)$;

$\mathrm{n}=\mathrm{a}$ intensidade de adsorção (NASCIMENTO et al., 2014).

Com os dados obtidos pelas isotermas de Langmuir e Freundlich para os radiotraçadores Co-57, Mn-54 e Zn-65, calcularam-se os parâmetros para a comparação das isotermas, de modo a verificar qual delas descreve o melhor comportamento dos metais em estudo.

Tabela 2 - Concentrações iniciais (pg. $\mathrm{L}^{-1}$ ) para Co-57, Mn-54 e Zn-65 utilizadas no procedimento experimental de determinação do $\mathrm{pH}$.

\begin{tabular}{c|c|c|c} 
pH & Co-57 & Mn-54 & Zn-65 \\
\hline 2,2 & 746 & 1,698 & 872 \\
\hline 3,0 & 738 & 1,687 & 855 \\
\hline 3,7 & 813 & 1,840 & 958 \\
\hline 4,8 & 765 & 1,734 & 893 \\
\hline 6,4 & 766 & 1,776 & 913 \\
\hline 6,8 & 757 & 1,734 & 895 \\
\hline 8,1 & 756 & 1,723 & 894 \\
\hline
\end{tabular}

Fonte: elaborada pelos autores (2019).

Tabela 3 - Concentrações iniciais (pg. L-1) para Co-57, Mn-54 e Zn-65 utilizadas no procedimento experimental de determinação do tempo de equilíbrio.

\begin{tabular}{c|c|c|c}
\hline Tempo (min.) & Co-57 & Mn-54 & Zn-65 \\
\hline 5 & 700 & 1,698 & 802 \\
\hline 10 & 718 & 1,670 & 815 \\
\hline 15 & 712 & 1,671 & 813 \\
\hline 20 & 703 & 1,644 & 810 \\
\hline 30 & 722 & 1,663 & 815 \\
\hline 60 & 723 & 1,698 & 829 \\
\hline
\end{tabular}

Fonte: elaborada pelos autores (2019)

Tabela 4 - Concentrações iniciais (pg. $\mathrm{L}^{-1}$ ) para Co-57, Mn-54 e Zn-65 utilizadas no procedimento experimental de variação das massas.

\begin{tabular}{c|c|c|c} 
Volume de solução mãe $(\mu \mathrm{L})$ & Co-57 & Zn-65 & Mn-54 \\
\hline 55 & 137 & 153 & 322 \\
\hline 110 & 296 & 328 & 708 \\
\hline 220 & 567 & 641 & 1,360 \\
\hline 440 & 1,128 & 1,267 & 2,716 \\
\hline 880 & 2,173 & 2,476 & 5,303 \\
\hline
\end{tabular}

Fonte: elaborada pelos autores (2019). 


\section{Caracterização do biocarvão}

A caracterização foi feita pelas técnicas de espectroscopia na região do infravermelho, análise termogravimétrica, microscopia eletrônica de varredura (MEV) e análise elementar. A espectroscopia na região do infravermelho do biocarvão foi realizada no espectrômetro VARIAN 660IR, operado na região de 4.000 a 600 $\mathrm{cm}^{-1}$, no Laboratório Multiusuário de Espectroscopia (LAME) da Universidade Federal Fluminense (UFF), permitindo a identificação dos grupos funcionais e tipos de ligações presentes na estrutura do biocarvão. Para a análise termogravimétrica do biocarvão, uma pequena quantidade dele foi inserida em um analisador térmico Neschtz, modelo Luxx STA 40, sob atmosfera de nitrogênio, no intervalo de temperatura entre $25^{\circ} \mathrm{e} 700^{\circ} \mathrm{C}$, com taxa de aquecimento igual a $20^{\circ} \mathrm{C}$ por minuto. Essa análise foi realizada no Laboratório 410 da UFF.

O equipamento utilizado para a MEV apresenta como fonte de elétrons um filamento de tungstênio (W) aquecido, e o sinal de imagem é gerado quando o feixe incidente interage com a superfície da amostra e é detectado, formando a imagem (DEDAVID; GOMES; MACHADO, 2007). O biocarvão foi submetido à metalização e, posteriormente, levado ao microscópio eletrônico de varredura (marca Jeol, modelo JSM 6510 LV) do laboratório de microscopia da Pontifícia Universidade Católica do Rio de Janeiro (PUC-RJ), a fim de gerar imagens de sua microestrutura para correlacioná-la à sua capacidade de adsorção. A análise elementar foi realizada no Laboratório de Eletroanalítica, Espectroanalítica e Análise Elementar Aplicada (LEEA) da PUC-RJ, com o uso do equipamento de análise elementar (Marca EA1112, Modelo LEEA 149). Este permitiu a determinação percentual de alguns elementos do biocarvão e, com base nesses teores, o cálculo do seu poder calorífico (HHV) pela Equação 5, em que: C, H, S, $\mathrm{O}$ e $\mathrm{N}$ são os percentuais de carbono, hidrogênio, enxofre, oxigênio e nitrogênio, respectivamente, e A é o percentual de cinzas (SOUZA; TEIXEIRA, 2015).

$\mathrm{HHV}=0,3491 \mathrm{C}+1,1783 \mathrm{H}+0,1005 \mathrm{~S}-0,1034 \mathrm{O}-0,0151 \mathrm{~N}-0,0211 \mathrm{~A}$

\section{RESULTADOS E DISCUSSÃO}

\section{Estudo das propriedades físico-químicas}

Considerando-se que as soluções comerciais de radiotraçadores possuíam alta atividades, foram realizadas diluições sucessivas de forma a obter uma solução mãe com radiotraçadores suficientes para realizar todos os experimentos e manter a melhor estatística nas contagens (BELLIDO; LATINI, 2014; SONDERMANN, 2015). Retirou-se então uma alíquota de $47 \mu \mathrm{L}$ da solução mãe, à qual se adicionaram 4,96 mL de água destilada em outro frasco acrílico, perfazendo o total de aproximadamente $5 \mathrm{~mL}$.

No procedimento experimental de determinação do $\mathrm{pH}$, foram obtidos teores percentuais de remoção dos radiotraçadores Co-57, Mn-54 e Zn-65 pelo biocarvão para cada solução de $\mathrm{pH}$. Com base nesses dados, foi construída a curva de eficiência (\%) de remoção dos radiotraçadores em função do $\mathrm{pH}$ do meio reacional (Figura 3).

Analisando-se o ajuste da curva de $\mathrm{pH}\left(\mathrm{R}^{2}=0,994\right)$ é possível concluir que, nos valores de $\mathrm{pH}$ entre 3,8 e 8,1, a curva tende a apresentar valores de eficiência (\%) de remoção constantes, em torno de $80 \%$. Isso indica a sua relevância para o presente estudo, tendo em vista que em ambientes naturais as águas se encontram nessa faixa de $\mathrm{pH}$, o que é favorável à adsorção (STUMM; MORGAN, 1996). Park et al. (2016) realizaram um estudo de biocarvão produzido com palha de gergelim em soluções de nitratos metálicos com pH 7. Boniolo et al. (2010) conduziram uma análise de biomassa residual de cascas de banana secas ao sol para a remoção de íons uranilo em soluções com pH 5. Amorim et al. (2016) investigaram o potencial de adsorção de chumbo II em conchas de pequi com soluções de pH 7. Pode-se observar na literatura que, comparativamente, a faixa de $\mathrm{pH}$ encontrada no presente estudo engloba os valores ótimos de $\mathrm{pH}$ de adsorção obtidos em trabalhos com diferentes materiais adsorventes (biocarvão e biomassa).

Com a faixa de $\mathrm{pH}$ de adsorção definida no experimento anterior, passou-se para os experimentos de determinação do tempo de equilíbrio da adsorção e de construção das isotermas de Freundlich e Langmuir. Estabeleceu-se um valor intermediário nessa faixa de $\mathrm{pH}$ preparando-se $5 \mathrm{~mL}$ de uma solução de $\mathrm{pH}$ 5,1, cujo preparo foi feito do modo já descrito na metodologia apresentada. Com o $\mathrm{pH}$ ideal de adsorção determinado e fixado ( $\mathrm{pH} 5,1$ ), foram obtidos os percentuais de remoção dos radiotraçadores Co-57, Mn-54 e Zn-65 pelo biocarvão, para os tempos de contato/agitação de 5, 10, 15, 20, 30 e 60 minutos. Os resultados obtidos estão apresentados na curva tempo em função da eficiência (\%) de remoção dos radiotraçadores (Figura 4), que mostra tendência constante de 20 minutos em diante.

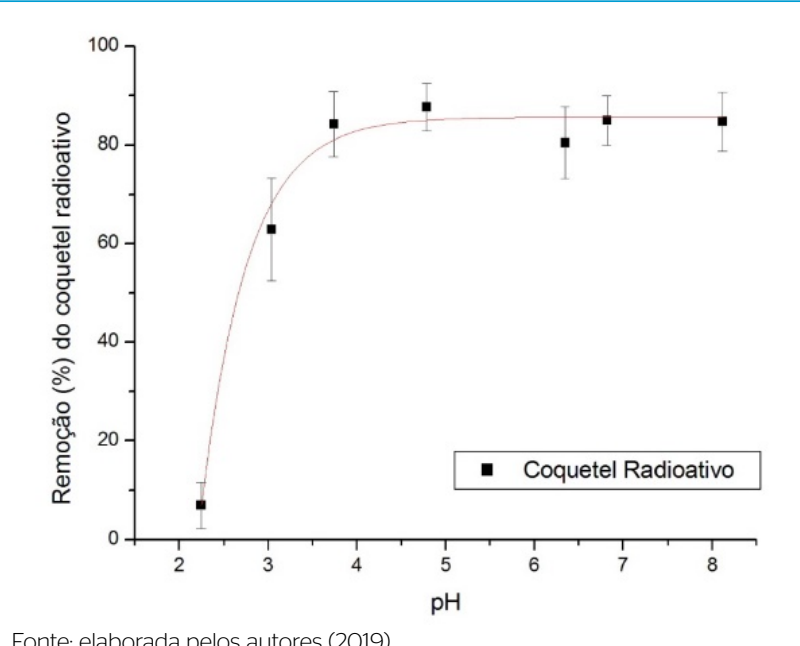

Fonte: elaborada pelos autores (2019)

Figura 3 - Curva de pH em função da eficiência (\%) de remoção dos radiotraçadores.

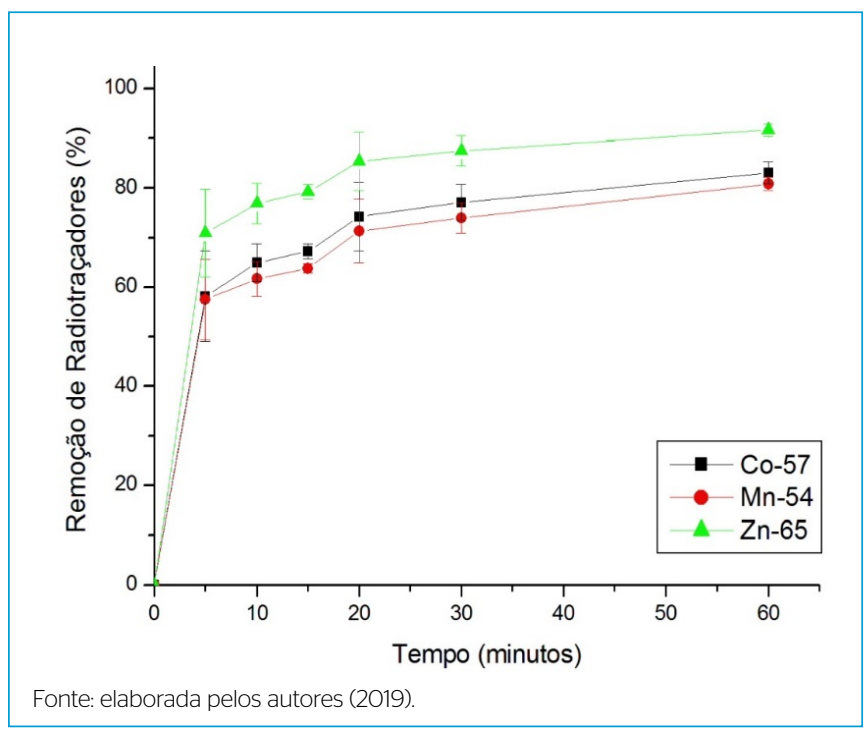

Figura 4 - Curva de tempo de contato/agitação em função da eficiência (\%) de remoção do coquetel radioativo. 
Considerando-se os pontos experimentais e as barras de erros, o tempo de equilíbrio escolhido para a realização do experimento de variação das massas (construção das isotermas de Freundlich e Langmuir) foi de 30 minutos. O tempo de 20 minutos está no ponto de inflexão da curva, e pequenos erros experimentais poderiam gerar baixos percentuais de remoção, não garantindo que o equilíbrio fosse atingido. Tendo em vista a proximidade dos percentuais de remoção para os tempos de 30 e 60 minutos, optou-se pelo de 30 minutos, otimizando assim o tempo gasto para a realização do experimento, o que também acarretou menor exposição à radiação, assegurando a aplicação das medidas de radioproteção.

Na literatura, observa-se uma diferenciação do tempo de equilíbrio, dependendo do material adsorvente e das condições em que ele foi produzido. Zhang et al. (2013) realizaram um estudo de adsorção de urânio em soluções aquosas, utilizando biocarvão produzido com biomassa de pinheiros por carbonização hidrotermal, em tempo ótimo de equilíbrio de 50 minutos, entretanto a curva experimental apresentou valores constantes para 20 minutos ou mais. Já no estudo de Amorim et al. (2016), por exemplo, para a avaliação do potencial de adsorção de chumbo II em conchas de pequi, foi obtida uma curva com valores de adsorção constantes para tempo de contato igual ou superior a 30 minutos, sendo este o escolhido pelos autores para a realização do experimento que, porventura, foi o mesmo adotado no presente trabalho.

Com o pH e o tempo de agitação/contato definidos, passou-se para a construção das isotermas. Para o experimento foram utilizadas diferentes atividades dos radiotraçadores, tendo sido preparados $5 \mathrm{~mL}$ de soluções com $\mathrm{pH}$ 5,1 e com alíquotas de 55, 110, 220, 440 e $880 \mu \mathrm{L}$ da solução mãe em duplicata para cada uma delas. Para manter o $\mathrm{pH}$ em 5,1, as soluções foram preparadas conforme descrito anteriormente. Após a realização dos experimentos, foram obtidos os valores de concentrações iniciais $\left(C_{o}\right)$ dos diferentes radiotraçadores antes do contato com o biocarvão ( $\left.g . \mathrm{L}^{-1}\right)$ e concentrações finais $\left(C_{e}\right)$ dos radiotraçadores na solução do filtrado após o contato com o biocarvão (g.L-1 $)$. Com esses dados, calculou-se a capacidade de adsorção (q) em g.g ${ }^{-1}$ e construíram-se as isotermas de Langmuir e Freundlich. A temperatura da solução de $\mathrm{pH}$ 5,1 , depois da agitação, foi de $21^{\circ} \mathrm{C}$.

Com base nos dados obtidos foi construída uma curva das diferentes alíquotas da solução mãe $(\mu \mathrm{L})$ em função da capacidade de adsorção q $\left(\mathrm{pg} \cdot \mathrm{g}^{-1}\right)$, para comparação entre as capacidades de adsorção dos três radiotraçadores pelo biocarvão $(q)$. As curvas resultantes estão representadas na Figura 5 e as equações e coeficientes de correlação $\left(\mathrm{R}^{2}\right)$ para a adsorção dos três radiotraçadores em estudo estão representados na Tabela 5.

A análise das curvas representadas na Figura 5 e dos parâmetros da Tabela 6 indicou proporcionalidade (coeficientes de correlação superiores a 0,99) entre a capacidade de adsorção e a massa de radiotraçador em contato com o biocarvão, ou seja, quanto maior a massa de radiotraçador, maior é a capacidade de adsorção do biocarvão em estudo. Esse comportamento apresenta-se linear na faixa de concentração utilizada no experimento, visto que as massas de radioisótopos empregadas nos experimentos são muito baixas, na ordem de grandeza de picogramas (Co-57 $<$ Zn-65 $<$ Mn-54).

As isotermas de Langmuir linearizadas, obtidas experimentalmente para a adsorção dos radiotraçadores pelo biocarvão de folhas secas de amendoeira-da-praia, são descritas nas Figuras 6A, 6b e 6C, enquanto as isotermas de Freundlich são descritas nas Figuras 6D, 6E e 6F.

Nas isotermas de Langmuir, os parâmetros constante de Langmuir $\left(\mathrm{K}_{\mathrm{L}}\right) \mathrm{e}$ capacidade máxima de adsorção $\left(\mathrm{q}_{\max }\right)$ foram definidos pelos coeficientes angulares e lineares, respectivamente. Nas isotermas de Freundlich, os parâmetros constante de equilíbrio de Freundlich $\left(\mathrm{K}_{\mathrm{F}}\right)$ e intensidade de adsorção $(\mathrm{n})$ foram definidos pelos coeficientes lineares e angulares, respectivamente. Os valores obtidos estão descritos na Tabela 6.

Os valores de $\mathrm{n}$ obtidos pela linearização das isotermas de Freundlich são todos maiores que 1, indicando um processo de adsorção favorável e a preferência dos radiotraçadores Zn-65, Co-57 e Mn-54 pela superfície do biocarvão.

Tabela 5 - Equações e coeficientes de correlação para comparação entre a capacidade de adsorção (pg.g $\mathrm{g}^{-1}$ ) dos radiotraçadores em função da variação da massa de radiotraçador, representada pelo volume de solução mãe $(\mu \mathrm{L})$.

\begin{tabular}{c|c|c} 
Radiotraçador & $\mathbf{R}^{2}$ & Equação \\
Co-57 & 0,999 & $\mathrm{q}=0,491 \mathrm{~V}+8,554$ \\
\hline Mn-54 & 0,997 & $\mathrm{q}=1,099 \mathrm{~V}+23,02$ \\
\hline Zn-65 & 0,998 & $\mathrm{q}=0,609 \mathrm{~V}+13,44$ \\
\hline
\end{tabular}

Fonte: elaborada pelos autores (2019).

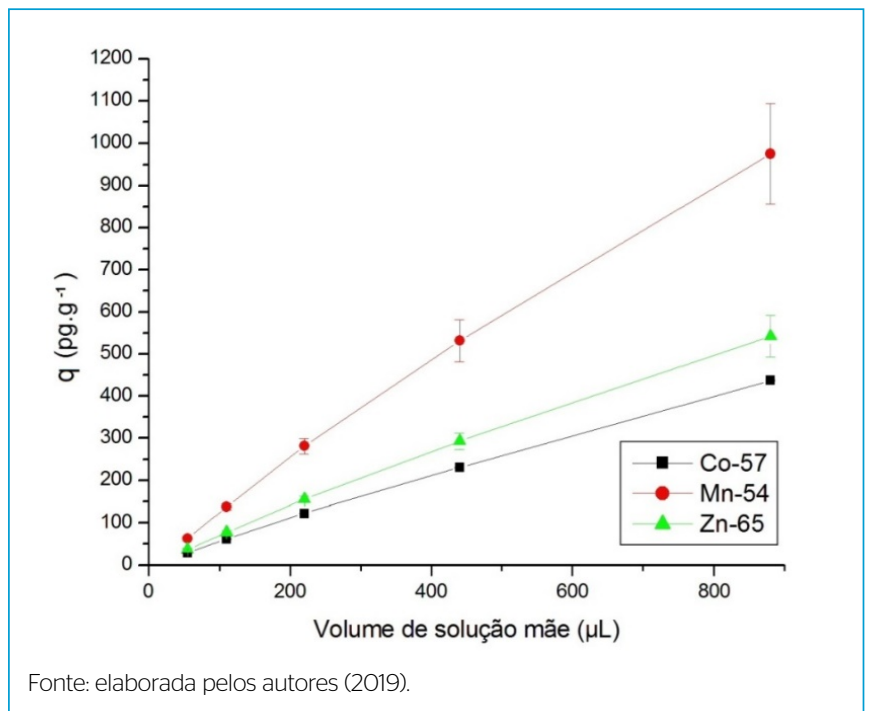

Figura 5 - Comparação entre a capacidade de adsorção (pg.g-1) de Co-57, Mn-54 e Zn-65 em função da variação da massa de radiotraçador, representada pelo volume de solução mãe ( $\mu \mathrm{L})$.

Tabela 6 - Parâmetros obtidos com o uso das isotermas linearizadas de Langmuir e Freundlich para a adsorção dos radiotraçadores Co-57, Mn-54 e Zn-65 pelo biocarvão de folhas de amendoeira-da-praia.

\begin{tabular}{|c|c|c|c|c|c|c|}
\hline \multirow{2}{*}{ Radiotraçador } & \multicolumn{3}{|c|}{ Parâmetros de Langmuir } & \multicolumn{3}{|c|}{ Parâmetros de Freundlich } \\
\hline & $\mathbf{R}$ & $\mathrm{K}_{\mathrm{L}}\left(10^{8}\right)$ & $\mathrm{Q}_{\text {máx }}\left(\mathrm{ng} \cdot \mathrm{g}^{-1}\right)$ & $\mathbf{R}$ & $\mathrm{K}_{\mathrm{F}}$ & $\mathrm{n}$ \\
\hline $\mathrm{Co}-57$ & 0,999 & 2,710 & 3,271 & 0,999 & 0,266 & 1,053 \\
\hline Mn-54 & 0,998 & 0,759 & 9,026 & 0,998 & 0,424 & 1,002 \\
\hline Zn-65 & 0,998 & 15,282 & 1,273 & 0,998 & 0,056 & 1,168 \\
\hline
\end{tabular}

Fonte: elaborada pelos autores (2019). 


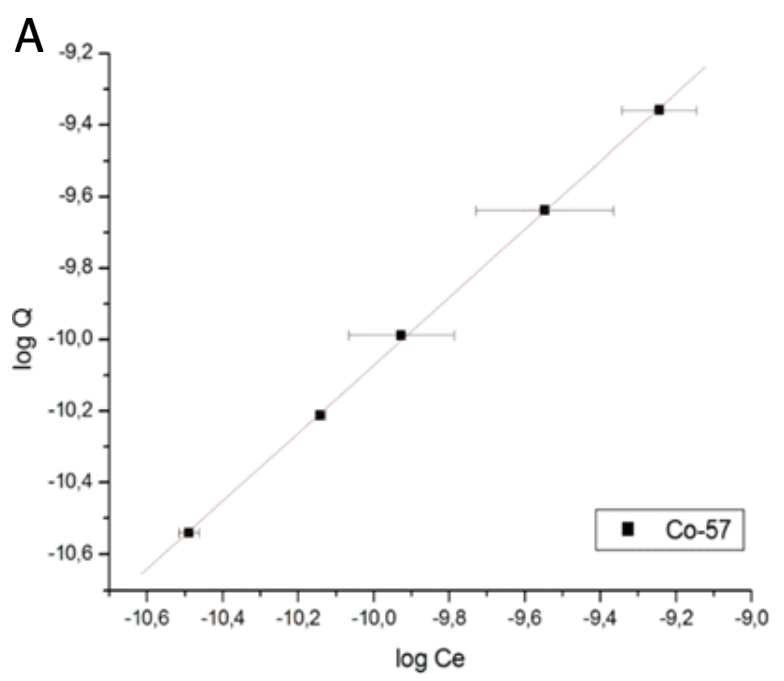

C

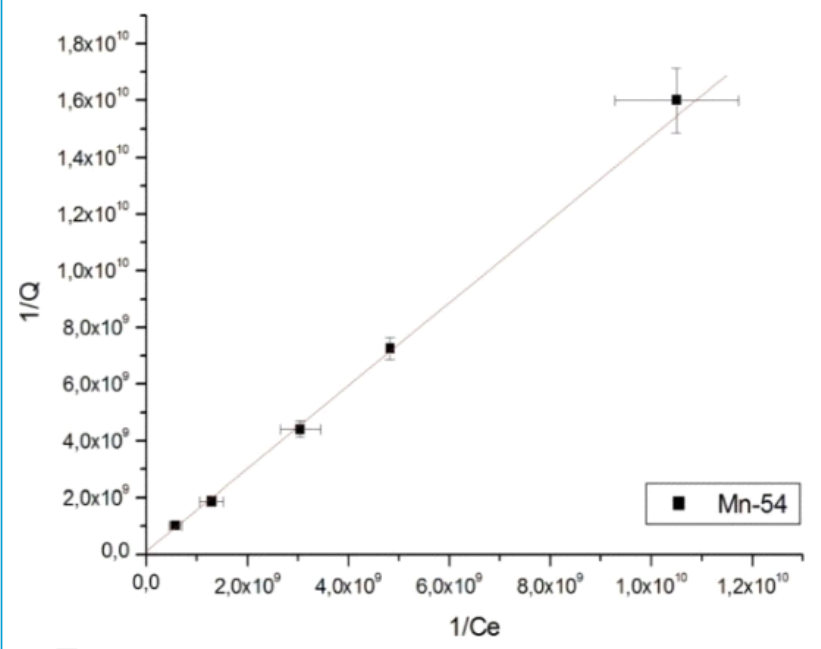

$\mathrm{E}$

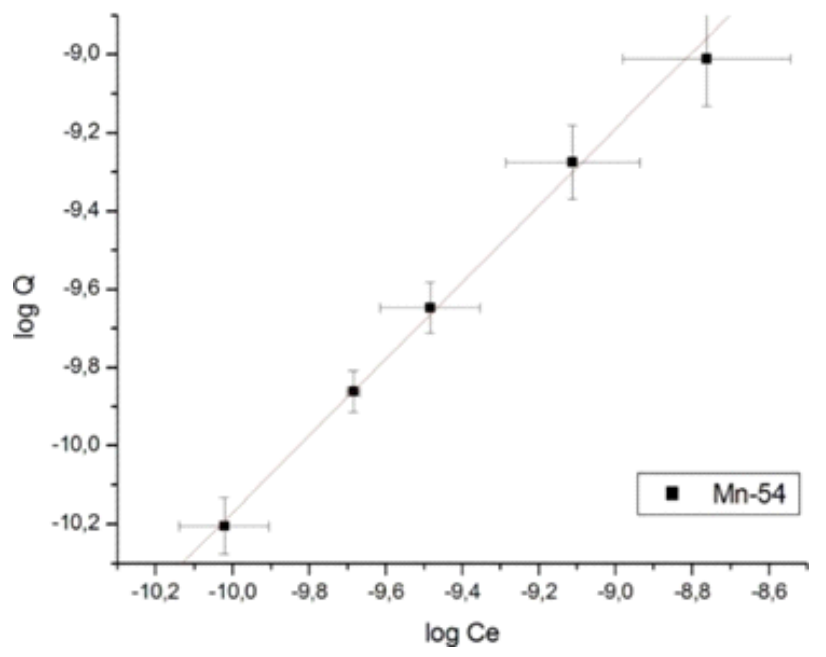

B

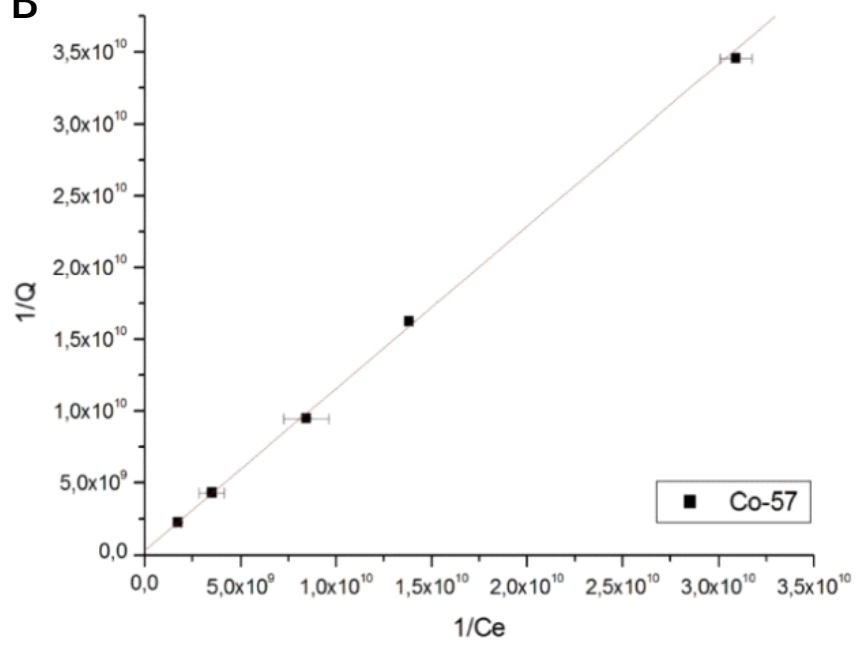

D

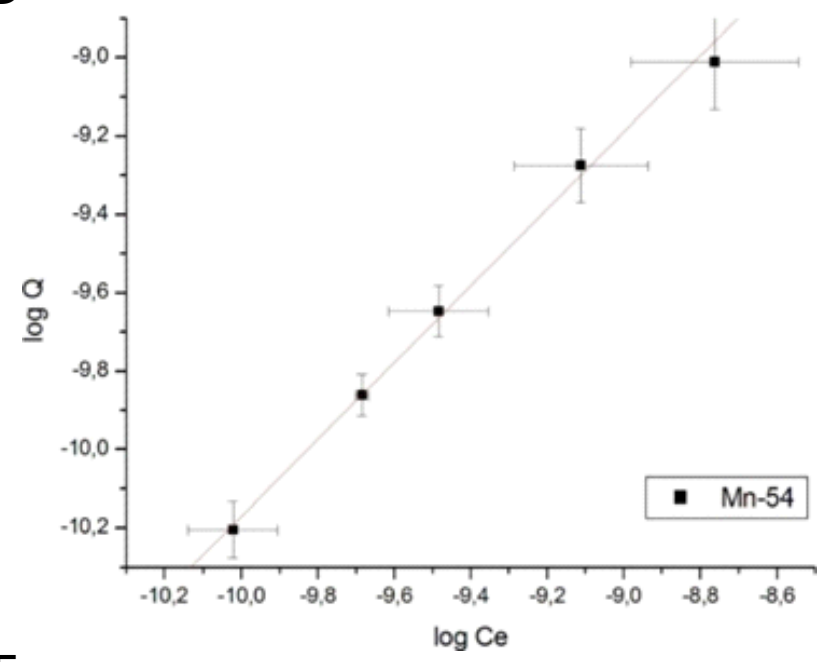

F

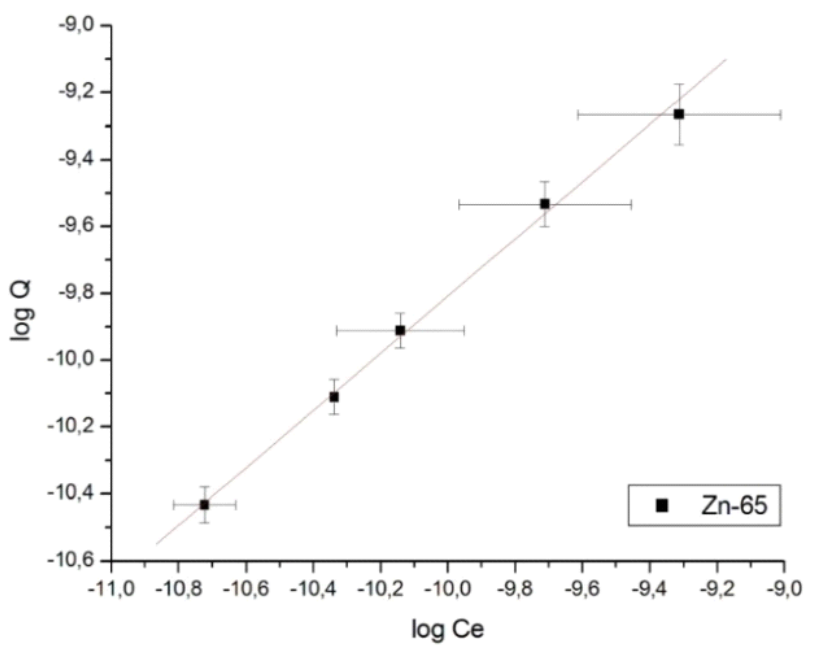

Fonte: elaborada pelos autores (2019).

Figura 6 - (A) Isoterma de Langmuir linearizada para adsorção de Co-57 pelo biocarvão em estudo. $R=0,999$ e equação dada por $1 / Q=1,128 \mathrm{I} / \mathrm{Ce}+3,057 \times 10^{8}$; $(B)$ isoterma de Langmuir linearizada para adsorção de Mn-54 pelo biocarvão em estudo. $R=0,998$ e equação dada por log $Q=1,4601 / C e+1,108 \times 10^{8}$; (C) isoterma de Langmuir linearizada para adsorção de Zn-65 pelo biocarvão em estudo. $R=0,998$ e equação dada por $1 / Q=0,514 \mathrm{I} / \mathrm{Ce}+7,855 \times 10^{8}$; (D) isoterma de Freundlich linearizada para adsorção de Co-57 pelo biocarvão em estudo. $R=0,999$ e equação dada por $\log Q=0,950 \log \mathrm{Ce}-0,575$; (E) isoterma de Freundlich linearizada para adsorção de Mn-54 pelo biocarvão em estudo. $R=0,998$ e equação dada por $\log Q=0,998 \log \mathrm{Ce}-0,373$; (F) isoterma de Freundlich linearizada para adsorção de $\mathrm{Zn}-65$ pelo biocarvão em estudo. $R=0,998$ e equação dada por $\log Q=0,856 \log \mathrm{Ce}-1,251$. 
Com as isotermas de Langmuir foi possível modelar a capacidade máxima de adsorção para os radiotraçadores: 1,273 ng.g ${ }^{-1}$ para o Zn-65, 3,271 ng.g ${ }^{-1}$ para o Co-57 e 9,026 ng.g ${ }^{-1}$ para o Mn-54. Levando-se em conta a competição pelos sítios ativos e o fato de os experimentos terem sido realizados com picogramas de radiotraçadores, já se vê uma capacidade de adsorção do biocarvão da ordem de $10^{3}$ vezes mais para todos os metais. Como mencionado, neste estudo foram utilizados os radiotraçadores na ordem de grandeza de picogramas e, portanto, ainda na faixa linear das isotermas, o que justifica os resultados de capacidades máximas de adsorção obtidos na ordem de nanogramas de radiotraçador por grama de biocarvão.

\section{Caracterização do biocarvão}

A análise mostrou que o biocarvão apresenta grupos funcionais característicos da celulose e lignina, componentes majoritários de sua biomassa (folhas secas de amendoeira-da-praia, Terminalia catappa), cujos comprimentos de onda $(\mathrm{nm})$ das bandas, seus respectivos grupos funcionais e modos vibracionais estão indicados na Figura 7.

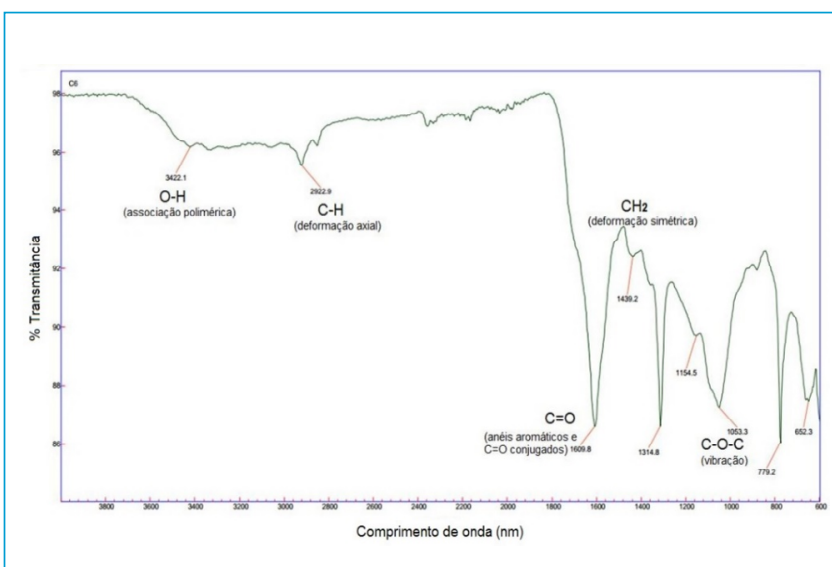

Fonte: elaborada pelos autores (2019).

Figura 7 - Espectro na região do infravermelho do biocarvão de folhas secas de amendoeira-da-praia, Terminalia catappa.
Os compostos da biomassa são constituídos principalmente de cadeias carbônicas condensadas de alta massa molar. O percentual de voláteis obtido foi de $9,47 \%$, enquanto o percentual de carbono fixo foi de $47,91 \%$. Tendo em vista que o biocarvão foi produzido em atmosfera de gás carbônico, observou-se baixo teor de voláteis, pelo fato de o gás carbônico ser mais oxidante que o nitrogênio, por exemplo. Em contrapartida, o teor de carbono fixo foi elevado, estando associado à presença de compostos de alto peso molecular, bem como aromáticos, o que também foi observado nas bandas identificadas na espectroscopia na região do infravermelho. Microscopicamente, visualizou-se que o biocarvão apresentou poros em forma de uma bolsa (Figura $8 \mathrm{~A}$ ). Além disso, escolheu-se um poro representativo da região observada e mediu-se o seu tamanho (Figura 8B), que foi de 4,2 $\mu \mathrm{m}$, sendo ele classificado como macroporo segundo a IUPAC (2015).

Os resultados indicaram que o biocarvão apresenta os percentuais de $48,8 \%$ de carbono, 3,3\% de hidrogênio, $1,0 \%$ de nitrogênio, menor que $0,3 \%$ de enxofre e 46,6\% de oxigênio, bem como o poder calorífico de $16,1 \mathrm{MJ} / \mathrm{kg}$.

\section{CONCLUSÕES}

O estudo das propriedades físico-químicas envolvidas em um processo de adsorção dos radiotraçadores Co-57, Mn-54 e Zn-65 pelo biocarvão produzido com uma biomassa de folhas secas de amendoeira-da-praia, Terminalia catappa, por conversão à baixa temperatura, indicou maior remoção de radiotraçadores para valores de $\mathrm{pH}$ de 3,8 ou maiores. O tempo de equilíbrio, por sua vez, foi estabelecido em 30 minutos ou mais, uma vez que os resultados percentuais de remoção tenderam a uma constante. Os parâmetros obtidos pelas isotermas de Freundlich indicam a preferência dos radiotraçadores pelo material adsorvente, no caso, o biocarvão. Com as isotermas de Langmuir foi possível modelar a capacidade máxima de adsorção dos metais estudados. $\mathrm{O}$ estudo com os radiotraçadores possibilitou conhecer bem a parte linear das isotermas, apontando para o potencial desse biocarvão como remediador em ambientes poluídos. Desse modo, as propriedades físico-químicas envolvidas no processo de adsorção deste estudo indicaram o potencial uso do biocarvão para a remediação de águas contaminadas com metais (cobalto, manganês e
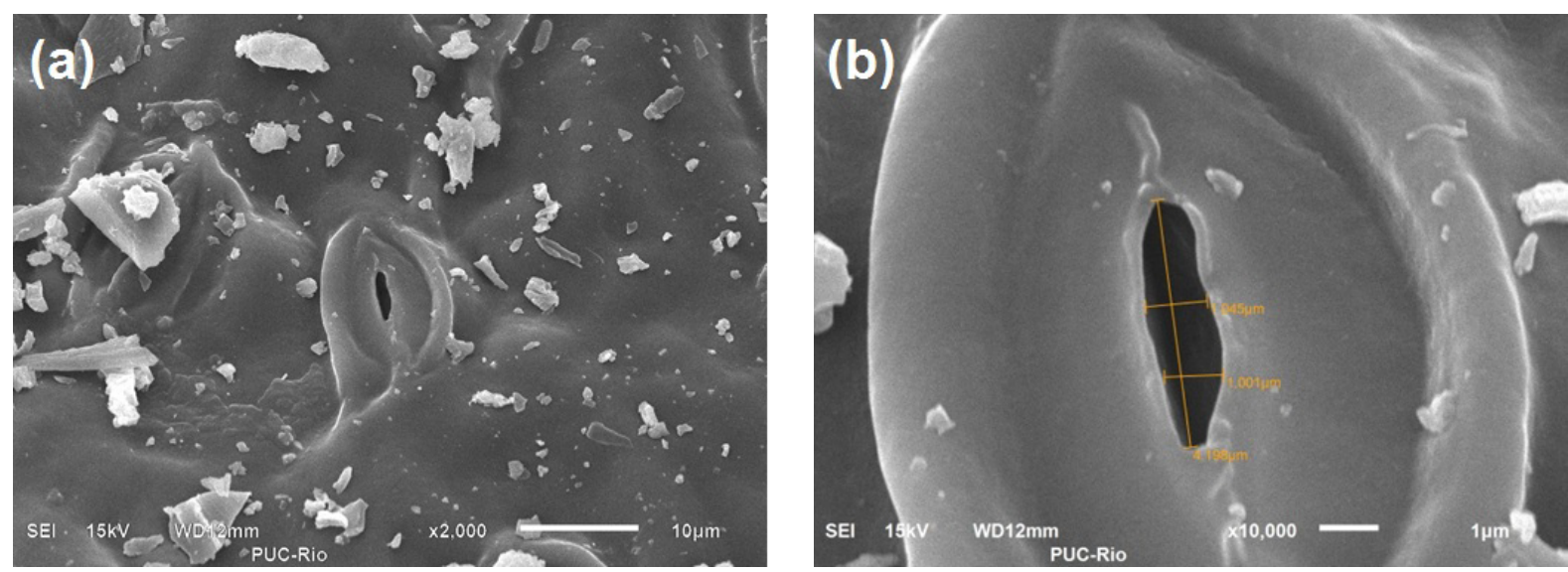

Figura 8 - Microscopia eletrônica de varredura biocarvão produzido com folhas secas de amendoeira-da-praia, Terminalia catappa, em atmosfera de gás carbônico. 
zinco), em caso de algum despejo inadequado ou acidente. Além disso, o biocarvão foi produzido por reutilização de folhas secas de amendoeira-da-praia, Terminalia catappa, garantindo o uso de técnicas ambientais mais sustentáveis.

\section{AGRADECIMENTOS}

No auxílio às medidas radiométricas, agradecemos à professora Carla Carvalho e equipe da LARAMAM/UFF.

\section{CONTRIBUIÇÕES DOS AUTORES}

Souza, C. C.: Curadoria de Dados, Investigação, Metodologia, Escrita — Primeira

Redação, Escrita - Revisão e Edição. Suzuki, K. N.: Metodologia, Supervisão, Escrita - Revisão e Edição. Latini, R. M.: Metodologia, Supervisão, Escrita Revisão e Edição. Canesin, F. P.: Metodologia, Supervisão, Escrita - Revisão e Edição. Teixeira, A. M. R. F.: Metodologia, Escrita - Revisão e Edição. Bellido, A. V. B.: Conceituação, Obtenção de Financiamento, Metodologia, Supervisão, Escrita - Revisão e Edição.

\section{REFERÊNCIAS}

AMORIM, D.J.; REZENDE, H.C.; OLIVEIRA, E.L.; ALMEIDA, I.L.S.; COELHO, N.M.M.; MATOS, T.N.; ARAÚJO, C.S.T. Characterization of Pequi (Caryocar brasiliense) Shells and Evaluation of Their Potential for the Adsorption of $\mathrm{Pb}^{\prime \prime}$ Ions in Aqueous Systems. Journal of the Brazilian Chemical Society, v. 27, n. 3, p. 616-623, 2016. https://doi.org/10.5935/0103-5053.20150304

BELLIDO, A.V.B.; LATINI, R.M. Apostila de Fundamentos de Química Nuclear. Niterói: Departamento de Físico-Química, Universidade Federal Fluminense, 2014. $116 \mathrm{p}$.

BELLIDO, A.V.B.; MACHADO, E.C.; CANESIN, F.P.; SUZUKI, K.N.; BERNEDO, L.F.B. Breve revisão sobre os estudos realizados com radiotraçadores artificiais para a obtenção de parâmetros físico-químicos em matrizes ambientais na floresta experimental de Itacuruçá/RJ. Geochimica Brasiliensis, v. 27, n. 2, p. 130-139, 2013. https://doi.org/10.21715/gb.v27i2.343

BONIOLO, M.R.; YAMAURA, M.; MONTEIRO, R.A. Biomassa residual para remoção de íons uranilo. Química Nova, v. 33, n. 3, p. 547-551, 2010. http:// doi.org/10.1590/SO100-40422010000300010

BRASIL. Resolução CONAMA no 420, de 28 de dezembro de 2009. Diário Oficial da União, Brasília, n. 249, p. 81-84, 2009

BRYAN, J.C. Introduction to Nuclear Science. $2^{\mathrm{a}}$ ed. Boca Raton: CRC Press Taylor \& Francis Group, 2013. 392 p.

DEDAVID, B.A.; GOMES, C.I.; MACHADO, G. Microscopia eletrônica de varredura: aplicações e preparação de amostras: materiais poliméricos, metálicos e semicondutores. Porto Alegre: EDIPUCRS, 2007.

INTERNATIONAL UNION OF PURE AND APPLIED CHEMISTRY (IUPAC). Physisorption of gases, with special reference to the evaluation of surface area and poro size distribution. Pure Applied Chemistry, v. 87, n. 9-10, p. 10511069, 2015. https://doi.org/10.1515/pac-2014-1117

KAWAI, B.; URIAS, C.; LEONEL, L.; ALMADO, M. Poluição ambiental por metais. 2017. Disponível em: http://www.fernandosantiago.com.br/met90. htm. Acesso em: 30 maio 2017.
KNOLL, G.F. Radiation Detection and Measurement. $4^{\mathrm{a}}$ ed. Nova York: John Wiley \& Sons, 2011. $864 p$

LABORATOIRE NATIONAL HENRI BECQUEREL (LNHB). Tables of evaluated data and comments on evaluation. LNHB, 2016. Disponível em: http://www. nucleide.org/DDEP_WG/DDEPdata.htm. Acesso em: 8 set. 2016.

NASCIMENTO, R.F.; LIMA, A.C.A.; VIDAL, C.B.; MELO, D.Q.; RAULINO, G.S.C. Adsorção: Aspectos teóricos e aplicações ambientais. Fortaleza: Imprensa Universitária da Universidade Federal do Ceará, 2014.

PARK, J.H.; OK, Y.S.; KIM, S.-H.; CHO, J.S.; HEO, J.S.; DELAUNE, R.D.; SEO, D. C. Competitive adsorption of heavy metals onto sesame straw biochar in aqueous solutions. Chemosphere, v. 142, p. 77-83, 2016. https://doi. org/10.1016/j.chemosphere.2015.05.093

RORIS, D.F.; COVRE, M.A.; COELHO, E.R.C. Estudo de adsorção do herbicida 2,4 D em carvões ativados destinados ao tratamento de água. In CONGRESSO BRASILEIRO DE QUÍMICA, 48., 2008. Anais [...]. Rio de Janeiro: Associação Brasileira de Química, 2008.

SONDERMANN, M.N. Estudos de processos físico-químicos com radiotraçadores em sedimentos costeiros para fins de biorremediação. Dissertação (Mestrado em Geociências) -Universidade Federal Fluminense Niterói, 2015

SOUZA, C.C.; TEIXEIRA, A.M. Obtenção de biocarvão por conversão a baixa temperatura a partir de rejeitos urbanos e estudo de sua influência em solos. Relatório Técnico (Iniciação Científica) - Fundação de Amparo à Pesquisa do Estado do Rio de Janeiro, Rio de Janeiro, 2015.

STUMM, W.; MORGAN, J.J. Aquatic Chemistry: Chemical Equillibria and Rates in Natural Waters. Noca York: John Wiley \& Sons, 1996. 1022 p.

Zhang, Z.-B.; Cao, X.-H., Liang, P.; LIU, Y.-H. Adsorption of uranium from aqueous solution using biochar produced by hydrothermal carbonization. Journal of Radioanalytical and Nuclear Chemistry, v. 295, n. 2, p. 1201-1208, 2013. 